\title{
Características clínicas y epidemiológicas de las toxicodermias en pacientes hospitalizados del Hospital Pablo Tobón Uribe, Medellín, Colombia, 2007-2009
}

\author{
María Cristina Trujilloº Luz Adriana Vásquez $^{2}$ \\ 1. Médica dermatóloga, Universidad Pontificia Bolivariana; docente, Universidad Pontificia Bolivariana, Medellín, Colombia \\ 2. Médica dermatóloga, Universidad Pontificia Bolivariana; coordinadora, Unidad de Dermatología, Hospital Pablo Tobón Uribe; docente, Universidad \\ Pontificia Bolivariana, Medellín, Colombia
}

\section{Resumen}

Las reacciones cutáneas a medicamentos, o toxicodermias, son una complicación frecuente e importante en la práctica médica. Hasta el momento, en Medellín (Colombia), no existen estudios ni publicaciones sobre el comportamiento de las reacciones cutáneas a medicamentos.

Objetivo. Describir las características clínicas y epidemiológicas de las reacciones cutáneas a medicamentos en los pacientes hospitalizados en el Hospital Pablo Tobón Uribe.

Métodos. Se llevó a cabo un estudio observacional descriptivo retro-prospectivo, en pacientes hospitalizados que presentaron reacciones cutáneas a medicamentos, en el periodo comprendido entre el $1^{\circ}$ de enero del 2007 y el 15 de noviembre de 2009.

Resultados. Se incluyeron 169 pacientes (52,7 \% hombres y 47,3\% mujeres). El rango de edad de presentación de las reacciones cutáneas a medicamentos se registró entre los 8 meses de edad y los 97 años, con una media de $38,5 \pm 24,7$ años. Los antibióticos fueron los responsables de más de la mitad de los casos (54,4\%), seguidos por los analgésicos (22,5\%), los anticonvulsionantes $(8,9 \%)$ y otros $(8,9 \%)$. En $66,3 \%$ de los pacientes, se logró identificar un sólo medicamento como agente causal. Los patrones clínicos más comúnmente encontrados fueron el exantema máculo-papular $(76,3 \%)$ y la urticaria (13,6\%). El promedio de área de superficie corporal comprometida fue de 47,07 $\pm 25,9 \%$.El área corporal más afectada fue la cara anterior del tórax $(76,3 \%)$, seguida por los miembros superiores (70,4\%), los miembros inferiores $(68,6 \%)$ y la espalda $(60,9 \%)$. La cara y el cuello fueron las zonas menos afectadas, con 39,1\% y $26 \%$, respectivamente.

Conclusión. Se demostró un comportamiento de las reacciones cutáneas a medicamentos en los pacientes hospitalizados, muy similares a lo descrito en otros países, sustentado en la presentación clínica de tipo exantema, más común en tórax y extremidades; también, los agentes causantes más frecuentes fueron los antibióticos y los analgésicos.

Palabras Clave: erupción por medicamentos, exantema, preparaciones farmaceúticas, antibióticos, anticonvulsionantes, analgésicos, toxicidad por medicamentos.

\section{Correspondencia:}

María Cristina Trujillo

Email:

maria_crys@hotmail.com

Recibido: 18 de junio de 2012. Aceptado: 25 de agosto de 2012.

No se reportan conflictos de intereses. 


\section{Summary}

Cutaneous reactions to drugs are a common and important complication in medical practice where the dermatologist plays a key role. So far, in Medellin, Colombia, there are no studies or any publications about the behavior of drug eruptions.

Objective: The clinical and epidemiologic characteristics of drug eruptions were described in hospitalized patients at the Hospital Pablo Tobón Uribe.

Methods: An observational descriptive retro-prospective study was carried out in patients hospitalized in the Hospital Pablo Tobón Uribe who showed skin reactions to drugs in the period from January $1^{\text {st }}, 2007$, to November 15, 2009.

Results: The study included 169 patients (52.7\% male and $47.3 \%$ women). The age range of presentation of skin reactions to drugs were recorded from 8 months to 97 years old, with a mean of $38.5 \pm 24.7$ years old. Antibiotics were responsible for more than half of the cases $(54.4 \%)$, followed by analgesics (22.5\%), anticonvulsants (8.9\%) and others $8.9 \%$. In $66.3 \%$ of patients, a single drug was identified as the causative agent. The most commonly encountered clinical patterns were macula-papular rash $(76.3 \%)$ and urticaria (13.6\%). The average body surface area involved was $47.07 \% \pm 25.9$. The most affected body area was the anterior chest in $76.3 \%$, followed by the upper limbs in $70.4 \%$, $68.6 \%$ lower limbs and back $60.9 \%$. The face and neck were less affect, with $39.1 \%$ and $26 \%$, respectively.

Conclusion: This observational study showed a behavior of skin reactions to drugs in hospitalized patients of Hospital Pablo Tobón Uribe very similar to that reported in other countries supported by the clinical presentation type rash, most commonly in the thorax and limbs, and for being the most frequent causative agent antibiotics and analgesics.

KeYwords: Drug eruptions, exanthema, pharmaceutical preparations, antibacterial agents, anticonvulsants, analgesics, drug toxicity

\section{Introducción}

Las reacciones cutáneas a medicamentos, o toxicodermias, son una complicación frecuente e importante en la práctica médica. El dermatólogo juega un papel fundamental en la definición del patrón clínico, la identificación del agente causal, el diagnóstico, la prevención y el tratamiento. Aunque son pocos los datos existentes en la literatura científica mundial, la incidencia de reacciones adversas a medicamentos en pacientes hospitalizados es de $15,1 \%$, y las reacciones cutáneas a medicamentos corresponden a 1 a $3 \%$, lo cual lleva a un aumento en la morbimortalidad, la estancia hospitalaria y los $\operatorname{costos}^{1-3}$.

Entre los fármacos más implicados en estas reacciones se incluyen los antibióticos, principalmente las penicilinas, el trimetoprim-sulfametoxazol y las cefalosporinas. En segundo lugar, se involucran los antiinflamatorios no esteroideos (AINE), anticonvulsionantes y medicamentos utilizados para quimioterapia, entre otros ${ }^{2,4}$. Factores como la edad, el sexo, la presencia de enfermedades subyacentes y la genética, pueden influir en el comportamiento y la incidencia de las toxicodermias.

Las toxicodermias son el motivo más frecuente de interconsultas solicitadas por otras especialidades a la Unidad de Dermatología, en el servicio de hospitalización del Hospital Pablo Tobón Uribe. Hasta el momento, en Medellín no existen estudios ni publicaciones sobre el comportamiento de las toxicodermias y es por esto que se pretende describir las características clínicas y epidemiológicas de las toxicodermias en los pacientes de este hospital.

\section{Materiales y métodos}

Se hizo un estudio observacional descriptivo retro-prospectivo en pacientes hospitalizados en el Hospital Pablo Tobón Uribe, que presentaron reacciones cutáneas o mu- 


\begin{tabular}{|cccc|}
\hline & Neoplasia & VIH & Exposición solar previa \\
& $n(\%)$ & $n(\%)$ & $n(\%)$ \\
\hline Sí & $27(16)$ & $7(4,1)$ & $6(3,6)$ \\
No & $142(84)$ & $162(95,9)$ & $156(92,3)$ \\
\hline Sin dato & o (o) & o (o) & $7(4,1)$ \\
\hline
\end{tabular}

TAbla 1. Presencia de neoplasias, VIH y antecedente de exposición solar.

\begin{tabular}{ccc}
\hline Medicamento & $\mathrm{n}$ & $\%$ \\
\hline Analgésicos & 38 & 22,5 \\
Antibióticos & 92 & 54,4 \\
Anticonvulsionantes & 15 & 8,9 \\
Antineoplásicos & 5 & 3,0 \\
Cardiovasculares & 3 & 1,8 \\
Otros & 15 & 8,9 \\
Sin dato & 1 & 0,6 \\
Total & 169 & 100,0 \\
\hline \hline
\end{tabular}

TABLA 2. Grupo de medicamentos causantes de reacciones cutáneas a medicamentos.

\begin{tabular}{ccc} 
Medicamento & $\mathrm{n}$ & $\%$ \\
\hline Analgésicos & 12 & 7,1 \\
Antibiótico & 34 & 20,1 \\
Anticonvulsionantes & 4 & 2,4 \\
Cardiovasculares & 1 & 0,6 \\
Otros & 6 & 3,6 \\
Sin dato & 112 & 66,3 \\
Total & 169 & 100,0 \\
\hline \hline
\end{tabular}

TABla 3. Segundo grupo de medicamentos implicados en la aparición de reacciones cutáneas a medicamentos. cosas a medicamentos, en el periodo comprendido entre el $1^{\circ}$ de enero del 2007 y el 15 de noviembre de 2009.

Se excluyeron los que recibieron transfusiones sanguíneas y sus derivados, e insumos y materiales médico-quirúrgicos liberadores de medicamentos durante la hospitalización.

$\mathrm{Al}$ evidenciar una reacción adversa a medicamentos en cualquier paciente hospitalizado, el médico tratante diligenciaba un formato de fármaco-vigilancia establecido por el hospital en la historia clínica electrónica. El Grupo de Fármaco-vigilancia del hospital era el encargado de informar al Servicio de Dermatología cuando la reacción adversa presentada tuviera compromiso de piel y mucosas. El dermatólogo encargado registraba todos los datos en un formulario prediseñado. La información se recolectó en una base de datos en Excel ${ }^{\mathrm{TM}}$, para luego ser analizada con SPSS ${ }^{\mathrm{TM}}$, versión 13.

Se analizaron la frecuencia absoluta y relativa de las variables cualitativas, y el promedio y la desviación estándar de las variables cuantitativas.

Este trabajo es una investigación descriptiva sin ningún riesgo para los pacientes. Se obtuvo la aprobación del hospital para examinar los pacientes, revisar las historias clínicas y completar el formulario. Toda la información recolectada se consideró de carácter confidencial. La información que permitiera identificar al paciente que participó en el estudio, fue manejada con absoluta reserva.

\section{Resultados}

En el estudio se incluyeron 169 pacientes (52,7 \% hombres y el 47,3\% mujeres) hospitalizados en el Hospital Pablo Tobón Uribe, durante el periodo del $1^{\circ}$ enero de 2007 al 15 noviembre de 2009.

El rango de edad de presentación de las reacciones cutáneas a medicamentos se registró entre los 8 meses de edad y los 97 años, con una media de $38,5 \pm 24,7$ años. El $24,9 \%$ de los casos reportados eran menores de 18 años y el 31,6 \%, mayores de 60 años. Los pacientes con piel de fototipo III fueron los más frecuentemente afectados $(44,4 \%)$, seguidos de aquellos con fototipo IV $(27,8 \%)$, fototipo II (13 \%), fototipo V (2,4 \%), fototipo I $(1,8 \%)$ y fototipo VI (o,6 \%). El porcentaje y el número de los pacientes con presencia de neoplasias asociadas, VIH y exposición solar previa, se presentan en la TABLA 1.

La vía de administración más utilizada en los que presentaron reacciones cutáneas fue la intravenosa (74\%), seguida de la oral $(25,4 \%)$ y la intramuscular (o,6 \%).

Entre los medicamentos implicados en la aparición de lesiones en piel, los antibióticos fueron responsables de más de la mitad de los casos $(54,4 \%)$. El segundo lugar fue ocupado por los analgésicos (22,5\%), seguidos por los anticonvulsionantes $(8,9 \%)$ y otros $(8,9 \%)$ (TaBLa 2). En 66,3\% de los pacientes, se logró identificar un sólo medicamento como agente causal. En los restantes (33,7\%), un segundo medicamento 
estuvo implicado y, entre ellos, el 20,7 \% correspondió a antibióticos, el 7,1 \% a analgésicos y el 2,4 \% a anticonvulsionantes (TABLA 3). Un tercer medicamento fue encontrado como posible fuente de la reacción cutánea en $5,3 \%$ de los pacientes.

De los medicamentos causantes de toxicodermias, se documentó que la dipirona y la vancomicina fueron los más reportados, con 17,2 \% y 10,1\% de los casos, respectivamente. El resto de los medicamentos estuvieron representados por piperacilina-tazobactam $(8,9 \%)$, ampicilina-sulbactam $(7,7 \%)$, ciprofloxacina $(5,9 \%)$, fenitoína (3,6 \%) y trimetoprim-sulfametoxazol, amoxacilina y carbamazepina, cada uno de ellos con 3,0 \% (TABLA 4). Al combinar los grupos de medicamentos sospechosos en un mismo paciente polimedicado, no se encontraron datos que sugirieran que una molécula unida a otra pudiera comportarse como causante de una reacción cutánea.

De los pacientes incluidos en el estudio, 129 (76,3\%) presentaron exantema máculo-papular, convirtiéndose este en el patrón clínico más comúnmente encontrado. De los pacientes, 23 (13,6\%) exhibieron inicialmente urticaria, seguida de eritrodermia en $7(4,1 \%)$, formas pustulares en $4(2,4 \%)$ y ampollosas en $3(1,8 \%)$. El o,6\% (un caso) correspondió a un síndrome de StevensJohnson y otro $(0,6 \%)$ presentó una vasculitis de pequeños vasos. No se documentaron otras formas clínicas, como necrólisis epidérmica tóxica, ni erupción fija por medicamentos.

El tiempo entre la administración del fármaco y la aparición de los síntomas estuvo entre 8 y 72 horas en $57(33,7 \%)$ de los pacientes; entre 5 y 30 días, en 35 $(20,7 \%)$; fue menor de una hora en $31(18,3 \%)$; estuvo entre 1 y 8 horas, en 22 (13,0\%); entre 72 horas y 7 días, en $8(4,7 \%)$, y fue más de 30 días en $3(1,8 \%)$. En 13 pacientes $(7,7 \%)$ el dato no fue reportado.

$\mathrm{Al}$ evaluar el tiempo de evolución de las lesiones en piel, los resultados mostraron que 94 (55,6 \%) de los casos llevaban entre 8 y 72 horas con los signos clínicos al momento del diagnóstico, 32 (18,9\%), entre 7 y 30 días, $31(18,3 \%)$, entre 72 horas y 7 días, 4 (2,4\%), entre 1 y 8 horas, 4 (2,4\%), menos de una hora, y 2 (1, $2 \%)$, más de 30 días. En 1,2 \% de los casos, no se encontró la información.

El promedio de área de superficie corporal (según la regla de los 9) comprometida fue de 47,07 $\pm 25,9 \%$, con un rango entre 2 y $95 \%$. En 99,4 \% de los casos, las lesiones fueron múltiples y en el o,6 \% restante no se anotó el dato. El área corporal más afectada fue la cara anterior del tórax $(76,3 \%)$, seguida por los miembros superiores $(70,4 \%)$, los miembros inferiores $(68,6 \%)$ y la espalda $(60,9 \%)$. La cara y el cuello fueron las zonas anatómicas menos afectadas, con 39,1 \% y $26 \%$, respectivamente.

\begin{tabular}{|c|c|c|}
\hline Medicamento & $n$ & $\%$ \\
\hline Dipirona & 29 & 17,2 \\
\hline Vancomicina & 17 & 10,1 \\
\hline Piperacilina-tazobactam & 15 & 8,9 \\
\hline Otros medicamentos & 15 & 8,9 \\
\hline Ampicilina-sulbactam & 13 & 7,7 \\
\hline Ciprofloxacina & 10 & 5,9 \\
\hline Fenitoína & 6 & 3,6 \\
\hline Amoxicilina & 5 & 3,0 \\
\hline Trimetoprim-sulfametoxasol & 5 & 3,0 \\
\hline Antineoplásicos & 5 & 3,0 \\
\hline Carbamazepina & 5 & 3,0 \\
\hline Cefazolina & 4 & 2,4 \\
\hline Meropenem & 4 & 2,4 \\
\hline Clindamicina & 4 & 2,4 \\
\hline Otros antibióticos & 3 & 1,8 \\
\hline Aztreonam & 2 & 1,2 \\
\hline Amikacina & 2 & 1,2 \\
\hline Ácido valproico & 2 & 1,2 \\
\hline Ampicilina & 2 & 1,2 \\
\hline Otros anticonvulsionantes & 2 & 1,2 \\
\hline Enalapril & 2 & 1,2 \\
\hline Morfina & 2 & 1,2 \\
\hline Aspirina & 2 & 1,2 \\
\hline Ibuprofeno & 2 & 1,2 \\
\hline Levofloxacina & 1 & 0,6 \\
\hline Antirretrovirales & 1 & 0,6 \\
\hline Gentamicina & 1 & 0,6 \\
\hline Dicloxacilina & 1 & 0,6 \\
\hline Imipenem & 1 & 0,6 \\
\hline Cefalexina & 1 & 0,6 \\
\hline Meperidina & 1 & 0,6 \\
\hline Tramadol & 1 & 0,6 \\
\hline Amiodarona & 1 & 0,6 \\
\hline Otros AINE & 1 & 0,6 \\
\hline Sin dato & 1 & 0,6 \\
\hline Total & 169 & 100,0 \\
\hline
\end{tabular}

TABLA 4. Medicamentos causantes de reacciones cutáneas a medicamentos. 


\section{Discusión}

El nombre de "reacción adversa a medicamentos" fue definido por la Organización Mundial de la Salud como "cualquier efecto nocivo, no intencional e indeseado de un medicamento que ocurre a dosis utilizadas para prevención, diagnóstico o tratamiento de una enfermedad"5. Aunque en la literatura científica mundial los datos son poco exactos debido a que una gran parte de estas reacciones no son reportadas, en un metaanálisis entre 1966 y 1996, se reportó una incidencia de reacciones a medicamentos en pacientes hospitalizados de $15,1 \%$, siendo graves en el $6,7 \%{ }^{6}$.

Como se sabe, las reacciones adversas a medicamentos pueden comprometer cualquier órgano de la anatomía humana, pero se considera que entre $10 \mathrm{y}$ $30 \%$ de ellas comprometen la piel y han sido llamadas reacciones cutáneas a medicamentos. Estas pueden ocasionar molestias leves o, incluso, pueden poner en riesgo la vida del paciente.

La incidencia de las reacciones cutáneas a medicamentos en los pacientes hospitalizados varía entre $1 \mathrm{y}$ $3 \%$, considerando que estos pacientes toman en promedio entre 8 y 9 medicamentos ${ }^{2,3,7}$. Los antibióticos son responsables de $7 \%$ de las reacciones cutáneas a medicamentos que ocurren en los pacientes hospitalizados ${ }^{8}$.

En este estudio observacional retrospectivo y prospectivo, se destacaron algunos aspectos importantes: el exantema máculo-papular y la urticaria fueron los patrones clínicos más frecuentes $(76,3 \%$ y 13,6 \%, respectivamente), lo cual coincide con los datos de la literatura científica mundial (95\% y $5 \%$, respectivamente $)^{7}$. Las reacciones graves, como el síndrome de Stevens-Johnson fueron muy raras (o,6 \%), tal como lo muestran algunos estudios publicados que indican que una de cada 1.000 reacciones cutáneas a medicamentos pueden poner en peligro la vida del paciente ${ }^{9-11}$. En este trabajo no se presentaron muertes explicadas por reacciones cutáneas a medicamentos.

El rango de edad de presentación de estas reacciones varía entre 8 meses y los 97 años, lo que confirma los amplios intervalos de edad reportados en varios estudios. Sin embargo, el $75 \%$ de los pacientes que presentaron reacciones cutáneas a medicamentos eran mayores de 18 años y, el 31,6\%, mayores de 60 años, hallazgos comparables con los de la literatura científica mundial ${ }^{6,12}$.

Las toxicodermias son posiblemente más frecuentes en los ancianos, debido a la polifarmacia, a las múltiples enfermedades concomitantes y a las alteraciones en sus parámetros farmacocinéticos y farmacodinámicos, ocasionados por la disminución funcional de algunos órganos vitales como el riñón, entre otros ${ }^{13,14}$.

Aunque en la literatura científica se describe que las reacciones cutáneas a medicamentos son 1,05 y 1,63 veces más comunes en mujeres menores de 14 años y adultas, respectivamente, lo que se explica por el mayor consumo de medicamentos por parte de este sexo, las diferencias en el volumen de distribución del medicamento y los cambios hormonales, en comparación con los hombres, los resultados de este estudio sugieren pocas diferencias entre los dos sexos $(52,7 \%$ hombres y $47,3 \%$ mujeres) $)^{15,16}$.

En nuestro medio, el 44,4\% de los pacientes que presentaron un reacción cutánea a medicamentos tenían piel de fototipo III según la clasificación de Fitzpatrick, teniendo en cuenta que la gran mayoría de nuestra población pertenece a este fototipo.

En pocos trabajos se ha reportado la relación entre algunas condiciones médicas de base y el desarrollo de reacciones cutáneas y, por el momento, no existe una correlación entre el diagnóstico de base del paciente y su pronóstico con el desarrollo de lesiones en piel ocasionadas por medicamentos ${ }^{7}$. En este trabajo, el $16 \%$ de los pacientes tenía una neoplasia de base y el 4,1\% estaba contagiados por el virus de la inmunodeficiencia humana (VIH/sida); sin embargo, no es posible confirmar la relación exacta entre la aparición de toxicodermias y estas enfermedades, debido a la metodología y a los objetivos del estudio. En cuanto a la exposición solar previa a la reacción cutánea, no se consideró un factor precipitante clave para el desarrollo de las lesiones en piel.

Acorde a la literatura científica, los medicamentos comúnmente implicados en la aparición de reacciones cutáneas son, en primer lugar, los antibióticos, siendo la vancomicina el más frecuente; en segundo lugar, los analgésicos, donde la dipirona es responsable de la mayoría de las reacciones cutáneas, y, en tercer lugar, los anticonvulsionantes, principalmente la fenitoína y la carbamazepina. Otros medicamentos relacionados con la aparición de reacciones cutáneas a medicamentos en pacientes hospitalizados, incluyen diuréticos, opiáceos, antiplaquetarios, antihipertensivos, antidepresivos, hipolipemiantes, etc. ${ }^{17-19}$.

Uno de los problemas en la práctica diaria se presenta cuando el paciente está polimedicado, que es lo usual en los hospitalizados,lo que hace mucho más difícil distinguir el agente etiológico responsable de la reacción ${ }^{20}$. En este estudio, se encontró que el 33,7 \% tenía dos medicamentos sospechosos como causa y el 5,3 \% recibía tres medicamentos implicados en el cuadro clínico; esto llevó al dermatólogo a usar algunos datos de la historia clínica relacionados con el tiempo de evolución de las lesiones, la fecha de inicio de los medicamentos y la relación con la aparición del cuadro clínico, entre otros, para determinar el agente causal más relacionado. Al combinar los grupos de medicamentos sospechosos en 
el mismo paciente, no se encontraron datos que sugirieran que una molécula unida a otra pueda comportarse como desencadenante de una reacción cutánea.

La mayoría de las reacciones cutáneas a medicamentos se presentaron en el primer mes después del inicio del medicamento y, al momento del diagnóstico, el 55,6 \% de los casos llevaba entre 8 y 72 horas de evolución de aparición de las lesiones cutáneas, lo que resalta la importancia de un diagnóstico y tratamiento precoces que eviten la progresión a formas más graves de toxicodermia. No se presentaron lesiones únicas como patrón clínico y, generalmente, los pacientes tenían un importante porcentaje de superficie de área corporal comprometido, con $\mathrm{u}$ promedio de $47,07 \pm 25,9 \%$. Las lesiones se localizaron principalmente en el tronco y las extremidades, con menor tasa de compromiso de cara y cuello, de acuerdo con lo descrito en la literatura científica.

\section{Conclusiones}

Las reacciones cutáneas a medicamentos son una causa frecuente de consulta en la práctica dermatológica, convirtiéndose en un problema significativo en los pacientes hospitalizados y contribuyendo a su morbilidad y mortalidad. Este primer estudio observacional de reacciones cutáneas a medicamentos en el Hospital Pablo Tobón Uribe de Medellín, demuestra que estas reacciones en esta población se comportan de una forma similar a lo que esta descrito en otros países y continentes.

Con este estudio, se abren nuevas puertas para el desarrollo de nuevas investigaciones que busquen relacionar las manifestaciones en piel con enfermedades específicas, determinar interacciones medicamentosas que aumenten la aparición de reacciones cutáneas a medicamentos, costos, y aumento de morbilidad y mortalidad, entre otros.

\section{AgRADECIMIENTOS}

Los autores expresan sus agradecimientos a Camilo Maya, por su colaboración, orientación y asesoría en el análisis estadístico; al Grupo de Farmacovigilancia y al Grupo de Dermatología del Hospital Pablo Tobón Uribe; a Claudia Patricia Palacios,Dermatóloga de la Universidad Pontificia Bolivariana, y a todas aquellas personas que en una $u$ otra forma colaboraron en el presente trabajo.

\section{Referencias}

1. Gruchalla R. Clinical asessment of drug-induces disease. Lancet. 2000;356:1505-11.

2. Svensson C, Cowen E, Gaspari A. Cutaneous drug reactions. Pharmacol Rev. 2000;53:357-79.

3. Beltrani V. Drug hypersensitivity. Immunol Allergy Clin North Am. 1998;18:867-95.

4. Gruchalla R. Allergic disorders. J Allergy Clin Inmunol. 2003;111:548-59.

5. World Health Organization. International drug monitoring: the role of the hospital. Technical Report Series No. 425.Geneva, Switzerland: World Health Organization; 1966.

6. Lazarou J, Pomeranz B, Corey P. Incidence of adverse drug reactions in hospitalized patients: a meta-analysis of prospective studies. JAMA. 1998;279:1200-5.

7. Bigby M. Rates of cutaneous reactions to drugs. Arch Dermatol. 2001;137:765-70.

8. Bigby $\mathrm{M}$, Jick S, jick M, Amdt K. Drug-induced cutaneous reactions: A report from the Boston Collaborative Drug Surveillance Program on 15,438 consecutive inpatients, 1975 to 1982. JAMA. 1986;256:3358-63.

9. Roujeau JC, Stern RS. Severe adverse cutaneous reactions to drugs. N Engl J Med. 1994;331:1272-85.

10. Hunziker T, Künzi UP, Braunschweig S, Zehnder D, Hoigné R. Comprehensive Hospital Drug Monitoring (CHDM): Adverse skin reactions, a 20-year survey. Allergy. 1997;52:388-93.

11. Fiszenson AF, Auzerie V, Mahe E, Farinotti R, Durand SC, Crickx $\mathrm{B}$, et al. A 6-month prospective survey of cutaneous drug reactions in a hospital setting. Br J Dermatol. 2003;149:1018-22.

12. Sharma VK, Dhar S. Clinical pattern of cutaneous drug eruption among children and adolescents in North India. Ped Dermatol. 1995;12:178-83.

13. Leach S, Roy SS. Adverse drug reactions: An investigation on an acute geriatric ward. Age Ageing. 1986;15:241-6.

14. Davies EC, Green CF, Mottram DR, Pirmohamed M. Adverse drug reactions in hospitals: A narrative review. Curr Drug Saf. 2007;2:79-87.

15. Schwartz JB. The influence of sex on pharmacokinetics. Clin Pharmacokinet. 2003;42:107-21.

16. Drici MD, Clement N. Is gender a risk factor for adverse drug reactions?_Drug Saf. 2001;24:575-85.

17. Pirmohamed M, James S, Meakin S, Green C, Scott AK, Walley TJ, et al. Adverse drug reactions as a cause of admission to hospital: Prospective analysis of 18 820 patients. BMJ. 2004;329:15-9.

18. Beijer HJM, Blaey CJ. Hospitalizations caused by adverse drug reactions (ADR): A meta-analysis of observational studies. Pharm World Sci. 2002;24:46-54.

19. Suh DC, Woodall BS, Shin SK, Hermes-DeSantis. Clinical and economic impact of adverse drug reactions in hospitalized patients. Ann Pharmacother.2000;34:1373-9.

20. Stephens MD. The diagnosis of adverse medical events associated with drug treatment. Adverse Drug React Acute Poisoning Rev. 1987;1:1-35. 